\section{S32 CHILDHOOD WHEEZE PHENOTYPES CAN BE USEFULLY SUB-CLASSIFIED USING LONGITUDINAL LUNG FUNCTION AND ATOPIC SENSITISATION DATA. EVIDENCE FROM THE SOUTHAMPTON WOMEN'S SURVEY}

doi:10.1136/thoraxjnl-2012-202678.038

SA Collins, KC Pike, HM Inskip, KM Godfrey, G Roberts, JW Holloway, JS Lucas. Southampton University, Southampton, United Kingdom

Background Epidemiological phenotypes for childhood wheeze were first proposed by the Tucson Children's Respiratory Study (TCRS), describing four distinct phenotypes. A new, six phenotype, characterisation has recently been proposed by the Avon Longitudinal Study of Parents And Children (ALSPAC). No previous cohort has included 1 year atopic sensitisation data with infant lung function in their analysis.

Objectives To classify infant and 6 year lung function and allergic sensitisation data at 1,3 and 6 years from the Southampton Women's Survey (SWS) cohort according to the ALSPAC 6 class pheno type model. To contrast this with TCRS phenotypes to assess clinical and epidemiological utility.

Methods At 6 years, 926 children had assessment of respiratory symptoms. Spirometry was measured in 791 children, with exhaled nitric oxide $(n=589)$ and methacholine challenge $(n=234)$. At $5-14$ weeks of age 95 of these children had lung function measured. Symptom data on wheeze status obtained at $6 \mathrm{~m}, 12 \mathrm{~m}, 2 \mathrm{y}, 3 \mathrm{y}$ and $6 \mathrm{y}$ follow up classified children into groups proposed from analyses of ALSPAC (never, early, transient, intermediate-onset, late-onset and persistent wheeze).

Results Persistent and intermediate-onset wheeze were significantly associated with atopy at 1, 3 and 6 years, and exhaled nitric oxide at 6 years. Late-onset wheeze was not associated with atopic sensitisation until 3 years. Persistent wheezers had lower infant $\left(V^{\prime} \operatorname{maxFRC} \mathrm{p}<0.05\right)$ and 6 year lung function $\left(\mathrm{FEV}_{1}, \mathrm{FEV}_{1} /\right.$ FVC and $\left.\mathrm{FEF}_{25-75}, \mathrm{p}<0.05\right)$, whilst late- and intermediate-onsetwheezers showed no lung function deficits. Transient wheezers were non-atopic but showed persistent lung function deficits $\left(\mathrm{FEF}_{25}\right.$ ${ }_{75}, \mathrm{p}<0.05$ and $\mathrm{V}^{\prime}$ maxFRC, $\mathrm{p}<0.001$ ), except for those who wheezed only in the first year of life (early phenotype).

Conclusion The SWS cohort data maps well into the ALSPAC phenotype classification, demonstrating useful subdivision of TCRS wheeze phenotypes. Lung function and atopy successfully differentiate persistent, late-onset and intermediate-onset wheeze, whilst the classical 'transient early' wheeze phenotype can be sub-classified into groups that reflect early lung function. This has potential significance for research into childhood wheeze and long term respiratory morbidity of children in these phenotypes.

\section{S33 VALIDATION OF THE GLI-2012 MULTI-ETHNIC SPIROMETRY REFERENCE EQUATIONS IN LONDON SCHOOL CHILDREN}

doi:10.1136/thoraxjnl-2012-202678.039

'S Lum, 'R Bonner, 12J Kirkby, ${ }^{12} S$ Sonnappa, '1 Stocks. 'Portex Respiratory Unit, UCL Institute of Child Health, London, UK; ${ }^{2}$ Great Ormond Streeet Hospital for Children NHS Foundation Trust, London, UK

The Global Lung Function Initiative (GLI) recently published the first global all-age (3-95 years) multi-ethnic reference equations for spirometry (Quanjer 2012 ERJ).

Aim To assess the agreement of the GLI reference range to contemporary lung function data from children living in Britain to verify its appropriateness for clinical use in this population.

Methods Anthropometry and spirometry were undertaken within a mobile laboratory in primary school children recruited to the Size and Lung function In Children study during one school year (2011-2012). Spirometry Z-scores were calculated using the GLI2012 reference range, using the 'Black equation' for South Asian children, for whom a specific equation has yet to be developed.
Results Spirometry data from 728 (47\% boys; 35\% White; 28\% Black; $26 \%$ South Asian; $11 \%$ other/mixed race) healthy children aged 5-11 years from eight London schools were eligible for inclusion. Mean (SD) age of 8 (1.6) years was similar across the 4 ethnic groups. After adjustment for age and sex, Black children were significantly heavier and taller compared to other groups, while South Asians were significantly lighter. When compared with White children, after adjusting for age, sex and standing height, $\mathrm{FEV}_{1}$ was significantly lower by a mean(95\%CI) of $14 \%(11 \%$; $16 \%)$ in Black; $11 \%(9 \% ; 14 \%)$ in South Asian and 4\% (1\%; $8 \%)$ in the "other"/ mixed race children. Similar patterns were seen for FVC but $\mathrm{FEV}_{1} /$ FVC was similar across all groups. When expressed according to ethnic-specific reference equations the mean (SD) for FVC, FEV and $\mathrm{FEV}_{1} / \mathrm{FVC}$ for the White, Black and other groups approximated 0 (1) suggesting that the GLI equations are appropriate for use in this population (Figure). Mean (95\%CI) FVC and FEV, were however $3 \%(-0.1 \%$; 6.1\%, $\mathrm{p}=0.07)$ higher in South Asian than Black children.

Conclusions This study shows that the GLI-2012 equations are appropriate for use in children across a wide range of ethnicities. Of the currently available equations, the 'Black equation' provides the best fit for South Asian children, although it results in slight overestimation of lung function, emphasising the need to develop specific coefficients for this group.

\section{S34 LUNG FUNCTION IN CHILDREN WITH SICKLE CELL DISEASE}

doi:10.1136/thoraxjnl-2012-202678.040

'J Kirkby, 'R Bonner, ${ }^{2 P}$ Bates, ${ }^{2} \mathrm{R}$ Strunk, 'F Kirkham, 'J Stocks, 'S Sonnappa. 'UCL, Institute of Child Health, London, UK; 'Washington University, Missouri, USA

Introduction Sickle Cell Disease (SCD) is one of the most prevalent genetic diseases with an incidence of $\sim 1$ in 200 Afro-Caribbean children in the UK (WHO; 2006). Since SCD can result in significant respiratory morbidity, ${ }^{[1]}$ lung function tests (LFTs) could play an important role in the clinical management of children with SCD.

Aim To determine the extent to which LFTs identify differences in children with SCD when compared with healthy Black children.

Methods A respiratory health questionnaire was administered, and four commercially available LFTs (Impulse oscillometry (IOS), specific effective airways resistance $\left(s R_{\text {eff }}\right.$ ), plethysmographic lung volumes, and spirometry) were undertaken in up to 214 healthy Black children and 85 children with SCD aged $4-12 y$.

Results Amongst children with SCD, 50\% reported cough on most days, and $25 \%$ had been reviewed by a specialist respiratory consultant within 3 months prior to the assessments. When compared with healthy children, $20 \%$ had a reduced total lung capacity (TLC), with concurrent reductions in $\mathrm{FEV}_{1}$ and $\mathrm{FVC}$ No differences in $\mathrm{sR}_{\text {eff }}$ were observed and IOS outcomes proved to be of limited value, due to poorly defined limits of normality and large between-subject variability. No significant group differences in bronchodilator responsiveness in SCD or healthy children were observed regardless of the outcome measured (Table 1).

Conclusion Despite the high proportion of respiratory symptoms reported, the number of children with LFTs falling outside the limits of normal was relatively small. Results suggest a pattern of restrictive lung disease in children with SCD. Of the outcomes assessed, baseline spirometry appears to be the most useful for routine assessment of lung disease in young children with SCD.

1. J Caboot, Curr Opin Pediatr 2008

2. Dencker, Clin Physiol Funct Imaging 2006

3. Kirkby, ERJ 2010

4. Kirkby, Ped Pulm 2012

5. Quanjer, ERJ 2012 


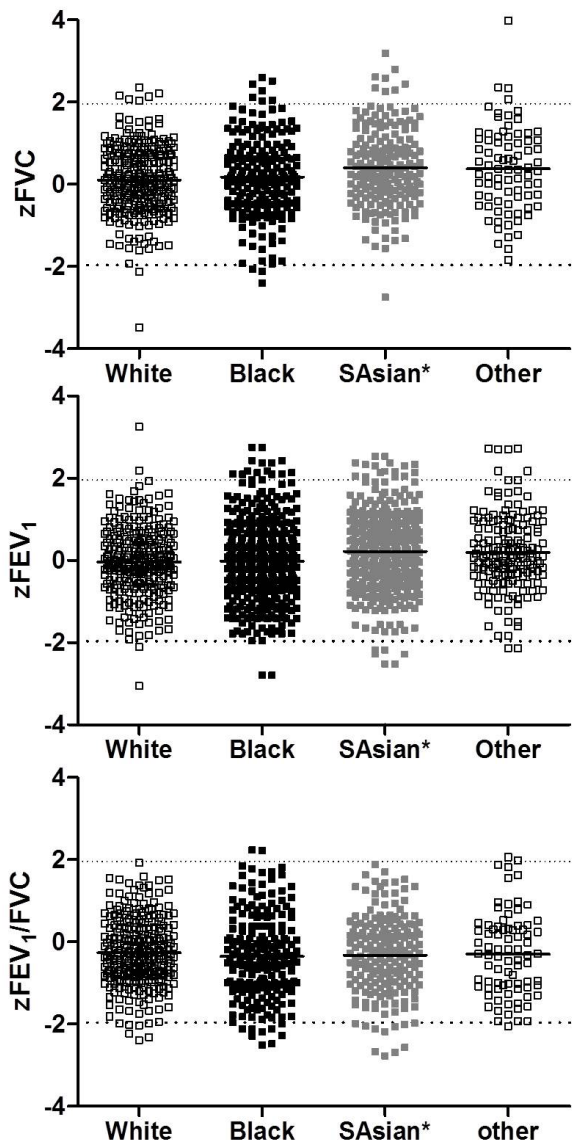

Figure: Lung function data according to the GLI2012 ethnic-specific reference equations. Data from South Asian children were expressed according to the 'Black' Equation. The "other" group comprise children of "mixed ethnic backgrounds" and those not covered by the other 3 groups.

Abstract S33 Figure 1

Abstract S34 Table 1 Comparison of Lung Function: Children with SCD vs. healthy Black controls.

\begin{tabular}{llc}
\hline & $\begin{array}{l}\text { Baseline Mean } \\
\text { CI) Diff (S5\% } \\
\text { Health) }\end{array}$ & $\begin{array}{l}\text { Bronchodilator Response } \\
\text { Mean (95\% CI) Diff } \\
\text { (SCD-Health) }\end{array}$ \\
\hline Resistance at 10Hz Z Score $^{[2]}$ & $-0.2(-0.4 ; 0.1)$ & $0.3(-0.2 ; 0.7)$ \\
SReff Z Score $^{[3]}$ & $-0.3(-0.6 ; 0.1)$ & $0.5(0.1 ; 0.8)$ \\
TLC Z Score & $-0.8(-1.1 ;-0.5)^{*}$ & \\
FVC Z Score & $-1.0(-1.2 ;-0.7)^{*}$ & $0.2(-0.1 ; 0.5)$ \\
FEV1 Z Score & $-1.2(-1.5 ;-0.9)^{*}$ & $0.2(-0.1 ; 0.5)$ \\
\hline
\end{tabular}

${ }^{*} p<0.001$. S35 DIFFERENT EARLY LIFE FACTORS ARE IMPORTANT IN THE
DEVELOPMENT OF ATOPIC AND NON-ATOPIC ASTHMA

doi:10.1136/thoraxjnl-2012-202678.041

${ }^{1} \mathrm{E}$ Petley, ${ }^{2} \mathrm{KC}$ Pike, ${ }^{3} \mathrm{HM}$ Inskip, ${ }^{4} \mathrm{KM}$ Godfrey, ${ }^{2} \mathrm{JSA}$ Lucas, ${ }^{4} \mathrm{G}$ Roberts, ${ }^{3}$ Southampton Women's Survey Study Group. 'Clinical and Experimental Sciences, University of
Southampton Faculty of Medicine, Southampton, UK; ${ }^{2}$ NIHR Southampton Respiratory Biomedical Research Unit, Southampton University Hospitals Trust, Southampton, UK; ${ }^{3}$ Southampton Medical Research Council Lifecourse Epidemiology Unit, University of Southampton, Southampton, UK; "Human DevelopmentHealth, University of Southampton Faculty of Medicine, Southampton, UK

Background Many factors have been related to the development of childhood asthma but there is inconsistency between studies.

Objective To understand how early life factors are linked to the development of the various asthma phenotypes at age 6 years in the Southampton Women's Survey (SWS) children's cohort.

Methods Data was collected from 940 children and their parents, primarily through questionnaires during pregnancy and at $6 \mathrm{~m}, 1,3$ and 6 years. Prevalent asthma was defined by a doctor's diagnosis and a wheezing episode in the last year. Data was analysed using STATA v9. A relative risk analysis using a univariate approach was undertaken, followed by a multivariate analysis.

Results Both maternal ( $R R=1.61, p=0.041)$ and paternal $(R R=2.05, p=0.002)$ atopic disease increased the risk of asthma at age 6 years. The risk increased with atopy, defined as a positive skin prick test, at 3 years $(R R=3.05, p<0.001)$ and with wheeze in the first 3 years $(R R=8.79, p<0.001)$. Episodes of bronchiolitis and chest infections were associated, in a dose-dependent manner, with the risk of asthma $(R R=1.50, p=0.022)$. Predictors in the multivariate model were wheeze in the first 3 years $(R R=10.74, p<0.001)$, atopy $(R R=2.87, \quad p<0.001)$ and maternal atopy $(R R=2.22$ $\mathrm{p}=0.011)$.When asthma at age 6 years was split into atopic and non-atopic asthma, the predictors were very different. Atopic asthma was associated with paternal atopy $(\mathrm{RR}=4.13, \mathrm{p}=0.002)$, male sex $(R R=2.56, p=0.030)$, atopy at 3 years $(R R=10.31$, $p<0.001)$ and wheeze in the first 3 years $(R R=5.91, p=0.004)$. In the multivariate analysis, the following were predictive: wheeze in the first 3 years $(R R=13.55, \quad p=0.012)$, atopy at 3 years $(R R=10.13,<0.001)$, paternal atopy $(R R=2.97, p=0.017)$ and a 12 month infant dietary pattern that follows current guidelines $(\mathrm{RR}=1.79, \mathrm{p}=0.016)$. For non-atopic asthma, bronchiolitis or chest infections $(R R=1.76, p=0.047)$, wheeze in the first 3 years $(R R=20.69, p=0.003)$ and tobacco smoke exposure at 6 years $(R R=2.16, p=0.035)$ increased the risk. Only wheeze in the first 3 years remained in the multivariate model.

Conclusions Different hereditary and early life factors modify the risk of atopic and non-atopic asthma at 6 years of age. This suggests that these two asthma phenotypes have different pathophysiologies.

\section{Pathophysiology of pulmonary vascular remodelling}

\section{S36 P38 MAPK: AN IMPORTANT PATHWAY IN THE PATHOBIOLOGY OF PULMONARY HYPERTENSION AND PULMONARY VASCULAR REMODELLING}

doi:10.1136/thoraxjnl-2012-202678.042

${ }^{1} \mathrm{AC}$ Church, ${ }^{2} \mathrm{R}$ Wadsworth, ${ }^{3} \mathrm{G}$ Bryson, ${ }^{3} \mathrm{DJ}$ Welsh, ${ }^{1} \mathrm{AJ}$ Peacock. ${ }^{1}$ Scottish Pulmonary vascular Unit, Glasgow, UK; 'University of Strathclyde, Glasgow, UK; 'University of Glasgow, Glasgow, UK

The p38 MAPK pathway is increasingly recognised as important in inflammation leading to systemic vascular disease but its role in pulmonary vascular disease is unclear. Our group has previously identified the p38MAPK $\alpha$ isoform to be critical in hypoxic-induced proliferation of pulmonary artery fibroblasts, a key step in the pathogenesis of pulmonary vascular remodelling. This study sought to investigate the role of p38MAPK in animal models of pulmonary hypertension and in human disease.

Methods Sprague Dawley rats were exposed to chronic hypoxia for 14 days and received a selective p38 MAPK $\alpha$ inhibitor from day 1 